\title{
B.E. Прохоров
}

\section{АКУСТИЧЕСКОЕ ИЗЛУЧЕНИЕ ПРИ СОУДАРЕНИИ КАПЛИ ПРОИЗВОЛЬНОЙ ФОРМЫ С ПОВЕРХНОСТЬЮ ВОДЫ}

\author{
Институт проблем механики им. А.Ю. Иилинского РАН \\ Россия, 119526, Москва, пр-т Вернадского, 101/1, \\ Тел.: 8(495) 434-60-63; 434-00-17,E-mail:prohorov@ipmnet.ru
}

\begin{abstract}
Рассматривается излучение высокочастотного звука в начальной стадии соударения капли с водной поверхностью в теоретической и экспериментальной постановке. Конечная формула ударного импульса содержит скорость соударения и текущие геометрические параметры донного сегмента, форма которого фиксируется на момент последнего перед столкновением видеокадра. Огибающая форма аппроксимируется непрерывной функцией, с помощью которой моделируется расширение горизонтального сечения донного сегмента с поверхностью и рассчитывается амплитуда ударного импульса. Результаты расчета удовлетворительно согласуются с данными акустических измерений.
\end{abstract}

Ключевые слова: капля, свободная поверхность, столкновение, звук, излучение, локальные параметры, моделирование, видеосъемка, гидрофон

При столкновении капли с водной поверхностью реализуются различные механизмы излучения звука: в начальной стадии доминирует ударная компонента, обусловленная сверхзвуковым расширение контактного контура, образуемым донным сегментом капли с поверхностью, а на последующих стадиях (десятки миллисекунд после контакта) - вступает в действие резонансное звучание воздушных пузырей, отрывающихся от подводных каверн $[1,2]$. Резонансное излучение крайне нестабильно, размеры и количество отрывающихся пузырей заметно разнятся от опыта к опыту, и в результате сильно различаются параметры излучаемых звуковых пакетов - частота, амплитуда, длительность [3].

Ударный импульс устойчиво появляется при каждом столкновении, при условии, что конечная скорость капли превышает пороговое значение, определяемое поверхностным натяжением и вязкостью [4].

Аналитическое описание ударного излучения построено на основе заимствований из теории, разработанной для капли/твердой сферы, соударяющейся с твердой/жидкой поверхностью [5-7]. Подход осно- 
ван на предположении о целостности капли в течение крайне короткой сверхзвуковой стадии сверхзвуковой стадии расширения.

Поскольку рассматривается капля правильной формы - сферической [5], или сфероидальной [6], то конечные формулы расчета давления радиус сферы или размеры полуосей сфероида.

Однако в реальности капля осциллирует по форме при падении. В серии повторяющихся опытов конечная форма меняется случайным образом, а сопутствующие ударные акустические импульсы столь же случайно меняются по амплитуде от опыта к опыту [8], без связи с известными теоретическими формулами.

В [4] показано, что теорию и эксперимент удается в какой-то мере согласовать, если вместо идеальных характерных размеров подставить локальные значения скорости и линейного размера на момент контакта. Для получения достаточной точности требуется высокая степень дискретизации контактной зоны на временном интервале порядка 10 нс. Однако инструменты, способные обеспечить такое разрешение, пока отсутствуют.

В настоящей работе измеренная штатными средствами дискретная последовательность координат профиля капли аппроксимируется непрерывной функцией, посредством которой моделируется движение профиля донного сегмента капли, зафиксированного в последнем кадре перед столкновением. Использование непрерывной функции позволяет восстановить радиус и скорость расширяющегося контактного контура с достаточным для сверхзвуковой стадии временным разрешением и вычислить ударное давление. Степень соответствия расчетных и экспериментальных значений ударного импульса свидетельствует об адекватности физической модели, в основе которой - целостность капли.

Рассматривается случай с осевой симметрией, когда ударное излучение принимается в точке на продолжении линии падения капли

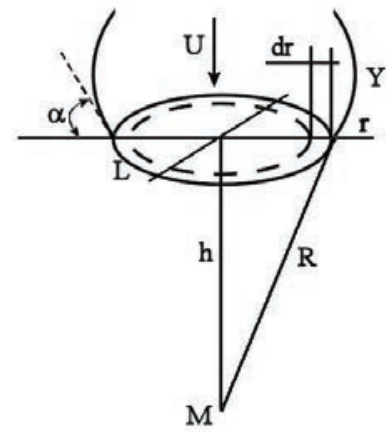
(рис. 1). Выбор простой геометрии позволяет провести экспериментальную проверку с помощью единственного гидрофона.

Рис. 1. Геометрия задачи при соударении капли с поверхностью. $Y$ - свободная поверхность капли, $L-$ контур сечения капли певерностью жидкости, $M$ - точка приема излучения. 
Водяная капля свободно падает на поверхность воды и сталкивается с ней на скорости $U$. Донный сегмент капли не разрушается при ударе и сохраняет целостность в пределах конечного, хотя и крайне малого, временного интервала, в течение которого его нижняя оконечность продолжает движение вглубь жидкости с постоянной скоростью U. Форма нижнего сегмента капли принимается фиксированной на момент съемки последнего перед приводнением кадра. Излучение звука при радиальном расширении контура $L$ со сверхзвуковой скоростью эквивалентно суммарному действию распределенных по контуру элементарных источников объемом $d V$ и плотностью $q$, так что каждый из них характеризуется объемной скоростью $d Q=q \cdot d V$. В данных переменных уравнение распространения звука выглядит [9]:

$$
\Delta p-\frac{1}{c^{2}} \frac{\partial^{2} p}{\partial t^{2}}=-\rho \frac{\partial q}{\partial t},
$$

стандартное решение которого:

$$
p(R, t)=\rho \frac{\partial}{\partial t} \int_{V} \frac{d Q(\tau)}{4 \pi R},
$$

где $\tau=t-R / c, c-$ скорость звука в воде. При заданной геометрии (рис. 1):

$$
\begin{gathered}
d Q=U \cdot d S=U \cdot 2 \pi r \cdot d r=2 \pi U R \cdot d R, \text { и условиях } h=\text { const }, h \gg r \\
p(R, t)=\frac{\rho}{2} \frac{d}{d t} \int_{h}^{R(\tau)} U \cdot d R=\frac{\rho U}{2} \frac{d R(\tau)}{d t} . \\
\text { Так как } r \cdot \dot{r}=R \cdot \dot{R}, \text { то } \frac{d R(\tau)^{h}}{d t}=\frac{r}{R} \frac{d r}{d \tau} \frac{d \tau}{d t} \approx \frac{r \dot{r}(\tau)}{h}\left(1-\frac{1}{c} \frac{d R(\tau)}{d t}\right), \text { откуда } \\
\frac{d R(\tau)}{d t}=\frac{\dot{r}}{h / r+\dot{r} / c} \text { и } \quad p(r, t)=\frac{\rho U \dot{r}}{2} \frac{1}{h / r+U_{e} / c} .
\end{gathered}
$$

В диапазоне естественных скоростей соударения, о которых речь в данной работе, справедливо:

$$
p(r, t)=\frac{\rho U \dot{r}}{2} \frac{r}{h}=\frac{\rho U^{2} r}{2 h} \operatorname{ctg} \alpha,
$$

учитывая очевидное соотношение: 


$$
r=U \operatorname{ctg} \alpha, \operatorname{ctg} \alpha=(d y / d r)^{-1}
$$

где $\alpha$ - угол между касательной к профилю вертикального сечения капли и осью $r$ (рис. 1).

В отличие от известных представлений $[5,6]$, где форма капли принимается фиксированной и фигурирует в условиях задачи через радиус сферы или полуоси сфероида, конечное выражение (3) записано в терминах локальных переменных, учитывающих наряду со скоростью текущие показатели размера $(r)$ и формы донного сегмента капли $(\operatorname{ctg} \alpha)$.

Радиус контура $y(t) r(t)$, ограничивающего окружность сечения капли поверхностью, определяется решением: $y(t)-U t=0$, где $y(t)-$ контур вертикального сечения донного сегмента, являющийся частью огибающей $Y(t)$ плоского изображения (видеокадра) капли. Огибающая $Y$ получена с помощью специальной компьютерной обработки, выделяющей на видеокадре высокоградиентную границу изображения капли. В качестве $y(t)$ выделялся небольшой участок огибающей, включающий точку контакта. Участок аппроксимировался аналитической функцией (полиномом 5-й степени), после чего моделировалось его движение вниз со скоростью $U$ на временном интервале 0.1-10 нс с шагом 0.1 нс. Таким образом, было получено 100 значений радиуса $r$ контактного контура, его временной производной $r$ и $\operatorname{ctg} \alpha$. По начальным значениям $r, \operatorname{ctg} \alpha$ и скорости приводнения $U$ (определялась по видеокадрам) вычислялся ударный импульс (3). Расчетное значение сравнивалось с измеренным давлением.

Капля воды отрывалась под собственным весом от сопла диаметром 4 мм и падала на водную поверхность.

Видеосъемка производилась камерой «Optronis CR3000x2» на скорости 10000 к/с с пространственным разрешением 0.04 мм. Ударный импульс измерялся гидрофоном ГИ800, погруженным на глубину $h$.

Текущий линейный размер и угол $\alpha$ заданы формой донного сегмента, которая уникальна в каждом опыте. В свою очередь, начальные значения $r$ и $\operatorname{ctg} \alpha$ вместе со скоростью столкновения $U$ определяют амплитуду $p_{m}$ ударного импульса (3). Последовательности форм, реализуемых в серии опытов, соответствует последовательность параметра: $\chi=U^{2} r \cdot \operatorname{ctg} \alpha$.

В соответствии с (3), при неизменной (в каждом опыте) форме, экспериментальная зависимость ударного давления от $\chi$ в серии опытов должна быть линейной.

На рис. 2 приведены амплитуды ударного давления, измеренные в повторяющихся опытах при падении капли с двух различающихся вы- 
сот. Прямая 1 получена согласно (3) подстановкой измеренной скорости приводнения $U$ и начальных значений $r$ и $\operatorname{ctg} \alpha=(d y / d r)^{-1}$, рассчитанных по профилю формы $Y$.

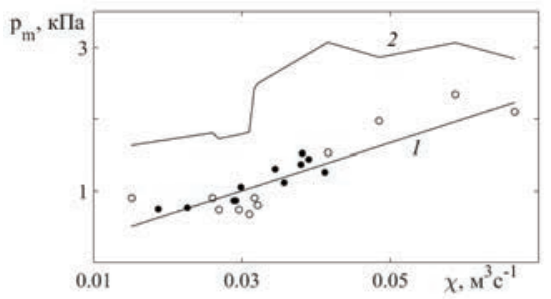

Рис. 2. Амплитуда рт ударного давления в повторяющемся опыте при фиксированной высоте отрыва капли в зависимости от $\chi=U^{2} r \times \operatorname{ctg} \alpha$. Эксперимент: $\mathrm{H}=112$ (точки) и 122 (кружки) см. 1 - теория [3], 2 - расчет по интегральным параметрам капли-сфероида [6].

Обе серии экспериментальных точек группируются вблизи теоретической зависимости (3), которая является линейной функцией аргумента $\chi=U^{2} r \times \operatorname{ctg} \alpha$ (рис. 2, прямая 1).

Разброс значений вызван в основном случайными смещениями рабочей точки АЧХ гидрофона из-за нестабильности спектра ударного импульса и погрешностью при фиксации момента приводнения капли. Среднее значение отклонения экспериментальных данных $p_{e}$ от прямой (3) составляет $\mu=\left\langle\left|p_{e}-p\right| / p_{e}\right\rangle=0.18$.

Кривая 2 на рис. 2 - амплитуда $P_{S}$ ударного давления, рассчитанная согласно $P_{S}=\rho U^{2} a^{2} b /(2 h)$ в приближении формы капли к правильным сфероидам [6], где $a$ и $b$ - горизонтальная и вертикальная полуоси капли, в качестве которых подставлены максимальные горизонтальный и вертикальный размеры, взятые из конечного видеокадра. Кривая 2 лежит значительно выше прямой 1 и реально измеренных данных, и это не только масштабное отличие - видно, что в поведении экспериментальных данных и кривой 2 мало подобия.

В экспериментах с изменяющейся высотой поведение скорости усложняется в еще большей степени (кривая 1, рис. 3a) - слабый рост скорости с высотой сопровождается колебаниями с размахом до $25 \%$ текущего значения. При этом скорость остается ниже гравитационной кривой $\sqrt{2 g H}$.

Несмотря на сложный характер изменчивости скорости и формы капли, экспериментальные данные ударного давления хорошо группируются вдоль зависимости $p(\chi)$ (прямая 1$)$ со средней величиной отклонения $\mu=0.15$. Нарастание амплитуды давления слабо коррелировано с ростом высоты отрыва $H$ - как высокие, так и низкие амплитуды давления распределены во всем диапазоне высот. Расчет по интегральным параметрам капли-сфероида [6] дает кривую 2, резко отличающуюся по амплитуде и форме от экспериментальной кривой. 


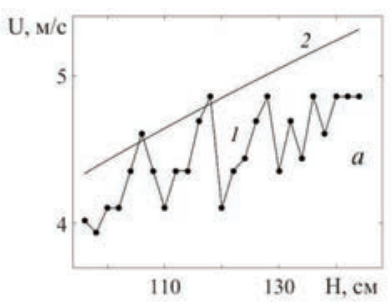

a

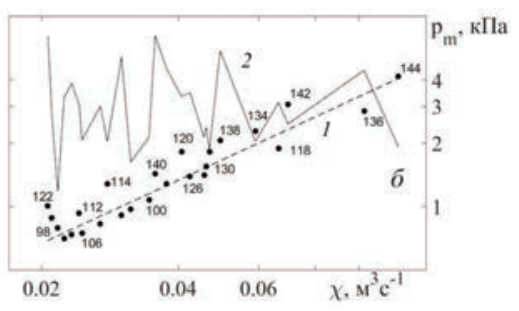

б

Рис. 3. а - Скорость приводнения капли в диапазоне высоты отрыва $H=96-144$ см, 1 - расчет по видеокадрам, $2-\sqrt{2 g H}, 6$ - зависимости амплитуды ударного импульса от $\chi=U^{2} r \times \operatorname{ctg} \alpha$ (масштаб - двойной логарифмический). Точки эксперимент (числа указывают выборочно высоту $H$ отрыва капли), 1 - теория

[3], 2 - расчет $P_{S}$ по интегральным параметрам капли-сфероида [6].

Форма капли, приобретаемая при подлете к водной поверхности, от опыта к опыту меняется случайным образом, однако в каждом опыте на стадии ударного излучения она сохраняет постоянство - об этом свидетельствует согласие расчетных и измеренных акустических данных.

Эксперименты выполнены на Гидрофизическом комплексе для моделирования гидродинамических процессов в окружающей среде и их воздействия на подводные технические объекты, а также распространения примесей в океане и атмосфере (ГФК ИПМех РАН).

Работа поддержана грантом РФФИ №18-05-0087.

\section{Литература}

1. Phillips S., Agarwal A., Jordan P. The Sound Produced by a Dripping Tap is Driven by Resonant Oscillations of an Entrapped Air Bubble// Sci Rep. 2018. Vol. 8. P. 9515.

2. Prosperetti A., Oguz H.N. The impact of drops on liquid surfaces and the underwater noise of rain // Annu. Rev. FluidMech. 1993. Vol. 25. P. 577-602.

3. Прохоров В.Е., Чашечкин Ю.Д. Излучение последовательности звуковых пакетов при падении капли на водную поверхность // ДАН. 2012. Т. 443. № 1. С. 48-52.

4. Прохоров В.Е. Влияние молекулярных эффектов на излучение звука при низкоскоростном столкновении капли с поверхностью воды // ЖЭТФ. 2018. Т. 153. № 4. С. 584-589.

5. Guo Y.P., Williams J.E. A theoretical study on drop impact sound and rain noise // J. Fluid Mech. 1991. Vol. 227. P. 345-355.

6. Howe M.S., Hagen N.A. On the impact noise of a drop falling on water // Journal of sound and vibration. 2011. Vol. 330. P. 625-635.

7. Field J.E. The physics of liquid impact, shock wave interactions with cavities, and the implications to shock wave lithotripsy // Phys. Med. Biol. 1991. Vol. 36. No. 1. P. 14751484.

8. Чашечкин Ю.Д., Прохоров В.Е. Структура первичного звукового сигнала при столкновении свободно падающей капли с поверхностью воды // ЖЭТФ. 2016. Т. 149. № 4. C. 864-875.

9. Morse P. Vibration and Sound. McGraw-Hill Book Company Inc., 1948. 


\title{
V.E. Prokhorov
}

\section{ACOUSTIC EMISSION IN THE COLLISION OF A DROP OF A RANDOM FORM WITH A WATER SURFACE}

\author{
Ishlinsky Institute for Problems in Mechanics RAS \\ Pr-ct Vernadskogo, 101/1, Moscow, Russia,119526, \\ Tel.: (495) 434-60-63; 434-00-17,E-mail:prohorov@ipmnet.ru
}

\begin{abstract}
Emission of high-frequency sound is considered in the initial stage of collision of a drop with a water surface in a theoretical and experimental setting. The final formula of the shock pulse contains the collision velocity and current geometric parameters of the bottom segment, the shape of which is fixed at the time of the last video frame before the collision. The envelope of the form is approximated by a continuous function, with the help of which the expansion of the horizontal section of the bottom segment by the surface is simulated and the amplitude of the shock pulse is calculated. The calculation results are in satisfactory agreement with the data of acoustic measurements.
\end{abstract}

Keywords: drop, free surface, collision, sound, radiation, local parameters, modeling, video filming, hydrophone

Acknowledgment: This work was supported by the RFBR grant No. 1805-0087. The work was supported by the RFBR grant No. 18-05-0087.

\section{References}

1. Phillips S., Agarwal A., and Jordan P. The Sound Produced by a Dripping Tap is Driven by Resonant Oscillations of an Entrapped Air Bubble. Sci Rep., Vol. 8, 9515.

2. Prosperetti A. and Oguz H.N. The impact of drops on liquid surfaces and the underwater noise of rain. Annu. Rev. FluidMech., 1993, Vol. 25, pp. 577-602.

3. Prokhorov V.E. and Chashechkin Yu.D. Emission of a sequence of sound packets when a drop falls on a water surface. DAN, 2012, Vol. 443, No. 1, pp. 48-52.

4. Prokhorov V.E. Influence of molecular effects on sound emission at low-speed collision of a drop with a water surface. ZhETF, 2018, Vol. 153, No. 4, pp. 584-589.

5. Guo Y.P. and Williams J.E. A theoretical study on drop impact sound and rain noise. J. Fluid Mech., 1991, Vol. 227, pp. 345-355.

6. Howe M.S. and Hagen N.A. On the impact noise of a drop falling on water. Journal of sound and vibration, 2011, Vol. 330, pp. 625-635.

7. Field J.E. The physics of liquid impact, shock wave interactions with cavities, and the implications to shock wave lithotripsy. Phys. Med. Biol., 1991, Vol. 36, No. 1, pp. 14751484.

8. Chashechkin Yu.D. and Prokhorov V.E. The structure of the primary sound signal when a freely falling drop collides with the water surface. ZhETF, 2016, Vol. 149, Vol. 4, pp. 864875.

9. Morse P. Vibration and Sound. FFI, McGraw-Hill Book Company Inc., 1948. 